\title{
Clinical importance of preoperative and postoperative prognostic nutritional index in patients with pancreatic ductal adenocarcinoma
}

\author{
Masahide Ikeguchi, Keisuke Goto, June Watanabe, Shoichi Urushibara, Tomohiro Osaki, \\ Kanenori Endo, Shigeru Tatebe, and Seiichi Nakamura
}

Department of Surgery, Tottori Prefectural Central Hospital, Tottori, Japan

\begin{abstract}
Backgrounds/Aims: The prognostic nutritional index (PNI) is based on the albumin concentration and absolute lymphocyte count and is designed to assess the nutritional and immunological status of patients. In this study, we evaluated the prognostic importance of the preoperative and postoperative PNI in patients who underwent curative resection of pancreatic ductal adenocarcinoma (PDAC). Methods: From 2006 to 2017, 50 patients with PDAC underwent curative resection at our hospital. We performed distal pancreatectomy (DP) with splenectomy in 15 patients, pancreaticoduodenectomy (PD) in 27 patients, PD combined with portal vein partial resection in 6 patients, and total pancreatectomy with splenectomy in 2 patients. We compared the preoperative PNI and postoperative PNI (1 and 2 months postoperatively) and analyzed the prognostic importance for these patients. Results: The mean PNI significantly decreased from 45.5 preoperatively to 39.8 at 1 month postoperatively $(p<0.001)$, but recovered to 42.7 at 2 months postoperatively. In 23 patients, the PNI at 2 months postoperatively recovered to the preoperative level (recovered group), but in the remaining 27 patients, the PNI at 2 months postoperatively did not reach the preoperative level (non-recovered group). The overall median survival time in the recovered group (29 months) was significantly longer than that in the non-recovered group (12 months, $p=0.003$ ). The multivariate overall analysis demonstrated that good recovery of the postoperative PNI was strongly correlated with a better prognosis. Conclusions: Effective postoperative nutrition may have a prognostic benefit for patients with operable PDAC. (Ann Hepatobiliary Pancreat Surg 2019;23:372-376)
\end{abstract}

Key Words: Overall survival; Pancreatic ductal adenocarcinoma; Prognostic nutritional index

\section{INTRODUCTION}

The prognostic nutritional index (PNI) is calculated using the following formula: $10 \times$ serum albumin concentration $(\mathrm{g} / \mathrm{dl})+0.005 \times$ lymphocyte count (number $/ \mathrm{mm}^{3}$ ) in the peripheral blood. ${ }^{1}$ Thus, this index reveals patients' immune status and nutritional condition. A low PNI indicates malnutrition, reduced neutrophil function, and reduced immunity. ${ }^{2,3}$ Additionally, a low preoperative PNI is reportedly associated with a high incidence of postoperative complications. ${ }^{4}$ Moreover, a significant correlation has been between a low preoperative PNI and poor survival of patients with various neoplasms. ${ }^{5-7}$ These reports indicate that the preoperative PNI may be a good prognostic indicator for patients with cancer.

The prognosis of patients with pancreatic ductal ad- enocarcinoma (PDAC) is extremely poor. Even if curative resection is performed, many patients die of cancer recurrence within a few months. Yamamoto et al. ${ }^{8}$ reported that the median survival duration of 195 patients who underwent pancreatic resection was 27.1 months. Additionally, Sato et al. ${ }^{9}$ reported that in patients who had undergone resection of PDAC in Japan, the estimated 5-year disease-specific survival rate was $23.1 \%$ and the diseasefree survival rate was $16.8 \%$. Moreover, postoperative complications such as anastomotic leakage between the pancreatic duct and the jejunum after pancreaticoduodenectomy (PD) or pancreatic fistula formation after distal pancreatectomy (DP) occur very frequently after resection of the pancreas. Thus, the nutritional status of many patients with PDAC is likely insufficient during treatment. However, changes in the postoperative PNI compared

Received: July 11, 2019; Accepted: July 11, 2019

Corresponding author: Masahide Ikeguchi

Department of Surgery, Tottori Prefectural Central Hospital, 730 Ezu, Tottori 680-0901, Japan Tel: +81-857-26-2271, Fax: +81-857-29-3227, E-mail: ikeguchim@tp-ch.jp

Copyright (C) 2019 by The Korean Association of Hepato-Biliary-Pancreatic Surgery

This is an Open Access article distributed under the terms of the Creative Commons Attribution Non-Commercial License (http://creativecommons.org/ licenses/by-nc/4.0) which permits unrestricted non-commercial use, distribution, and reproduction in any medium, provided the original work is properly cited. Annals of Hepato-Biliary-Pancreatic Surgery • pISSN: 2508-5778 - elSSN: 2508-5859 
with the preoperative level and the relationship between the postoperative PNI and patients' prognosis have not been thoroughly studied. Therefore, we evaluated the clinical importance of the preoperative and postoperative PNI as a prognostic marker in patients with PDAC.

\section{PATIENTS AND METHODS}

\section{Patients}

From 2006 to 2017, we enrolled 50 patients who were diagnosed with operable PDAC and underwent curative resection (no residual tumors macroscopically) at Tottori Prefectural Central Hospital. The patients' details are shown in Table 1. Clinical and pathological staging of PDACs was performed using the American Joint Committee on Cancer $6^{\text {th }}$ edition TNM staging system for pancreatic cancer. ${ }^{10}$ All patients were followed up at Tottori Prefectural Central Hospital until April 2019. No patients received chemotherapy or radiation therapy before the operation. Informed consent for medical treatment and use

Table 1. Sample characterization $(n=50)$

\begin{tabular}{lc}
\hline \multicolumn{1}{c}{ Parameters } \\
\hline Median age (years, range) & $72(37-88)$ \\
Gender (male/female) & $32 / 18$ \\
Median preoperative CA 19-9 levels & $196.1(1-2640)$ \\
(U/ml, range) & \\
Median preoperative PNI levels (range) & $46(27.8-57.6)$ \\
Operative procedures (\%) & $15(30)$ \\
DP & $27(54)$ \\
PD & $6(12)$ \\
PD combined with portal vein partial & \\
resection & $2(4)$ \\
Total pancreatectomy & $302(148-568)$ \\
Median operation time (minutes, range) & \\
Pathological tumor stages (\%) & $2(4)$ \\
IA & $1(2)$ \\
IB & $7(14)$ \\
IIA & $35(70)$ \\
IIB & $5(10)$ \\
III & \\
Postoperative complications & 30 \\
No & $20(7 / 3 / 10)$ \\
Yes (pancreatic fistula, passage & \\
disorder, others) & $25(10-238)$ \\
Median postoperative hospital stay & \\
(day, range) & \\
\hline CA & \\
\hline
\end{tabular}

CA 19-9, carbohydrate antigen 19-9; DP, distal pancreatectomy; $\mathrm{PD}$, pancreaticoduodenectomy; PNI, prognostic nutritional index of clinical data from the medical records was obtained from all patients.

\section{Surgical procedure}

All patients underwent open laparotomy. According to the tumor location, we performed DP with splenectomy in 15 patients, PD in 27 patients, PD combined with portal vein partial resection in 6 patients, and total pancreatectomy with splenectomy in 2 patients. Reconstruction after PD with or without partial resection of the portal vein was performed by Child's method. An internal short stent was placed across the pancreatojejunostomy, but no stent was placed following choledochojejunostomy.

\section{Postoperative complications}

Postoperative complications were analyzed by reviewing the patients' clinical data. The severity of postoperative complications was graded according to the Clavien Dindo classification. ${ }^{11}$ Clavien Dindo grade $\geq$ III complications were considered major complications.

\section{Clinical data}

We recorded clinical parameters including the operation time, postoperative hospital stay, and occurrence of postoperative complications. Blood samples were routinely taken from each patient at the time of admission to our hospital. The serum albumin concentration, total lymphocyte count, and level of the tumor marker (carbohydrate antigen 19-9, CA 19-9) were recorded. The reported reference range of the serum CA $19-9$ level is 0 to $37 \mathrm{U} / \mathrm{ml}^{12}$ Additionally, the PNI was calculated preoperatively and at 1 and 2 months postoperatively. This study was approved by the ethics review board of Tottori Prefectural Central Hospital (approval number: 2019 - 2).

\section{Statistical analysis}

Differences between two normally distributed parameters were compared using the $\chi^{2}$ test and Fisher's exact probability test. Wilcoxon's signed rank test was used to assess the correlation between the two numerical parameters (preoperative and postoperative PNI). The Mann Whitney $U$ test was used to compare differences between two parameters with non-normal distributions. Long-term overall survival (OS) was calculated using the KaplanMeier method, and the prognostic difference between the 
two groups was compared using the log-rank test. All data were analyzed by StatView software (Abacus Concepts, Inc., Berkeley, CA, USA). A $p$ value of $<0.05$ was considered statistically significant.

\section{RESULTS}

The mean PNI at 1 month postoperatively was 39.8 (range, 14-57.2), which was significantly lower than the preoperative PNI of 45.5 (range, 27.8-57.6; $p<0.001$ ). The mean PNI significantly recovered to 42.7 (range, 16.9-59.5) at 2 months postoperatively compared with that at 1 month postoperatively $(p=0.002)$. However, the PNI at 2 months postoperatively did not reach the preoperative level ( $p=0.045$, Fig. 1). In 23 patients, the PNI at 2 months postoperatively recovered to the preoperative level (recovered group), but in the remaining 27 patients, the PNI at 2 months postoperatively did not reach the preoperative level (non-recovered group). At 1 and 2 months postoperatively, the PNI in the recovered group was signi-

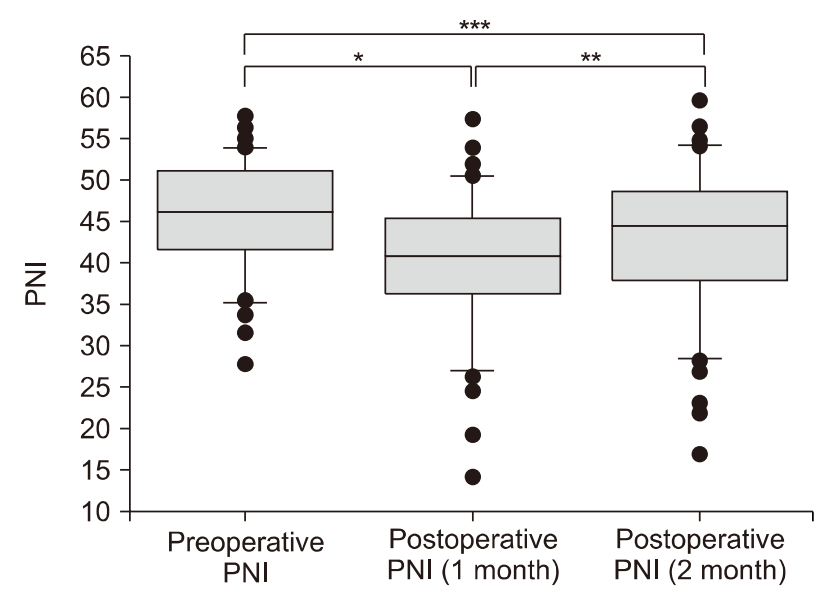

Fig. 1. The mean prognostic nutritional index was 45.5 (range, 27.8-57.6) preoperatively, 39.8 (range, 14.0-57.2) at 1 month postoperatively, and 42.7 (range, 16.9-59.5) at 2 months postoperatively. ${ }^{*} p<0.001,{ }^{* *} p=0.002, * * * p=0.045$. ficantly better than that in the non-recovered group $(p<$ 0.001, Table 2).

Major postoperative complications occurred in $40 \%$ of patients. One patient died of postoperative peritonitis secondary to leakage at the pancreatojejunostomy site 3 months postoperatively. Thus, the operative mortality rate in our series was $2 \%$. The mean postoperative hospital stay among the 20 patients with major postoperative complications (54.9 days) was significantly longer than that among the 30 patients without postoperative complications (21.6 days, $p<0.001)$. However, we found no significant correlation between the preoperative PNI and the occurrence of postoperative complications ( $p=0.751)$. Among the 27 patients in the non-recovered group, 22 underwent PD or total pancreatectomy $(81.5 \%)$. Although this proportion of patients was higher than that in the recovered group $(13 / 23,56.5 \%)$, the difference was not statistically significant $(p=0.055)$.

The 5-year OS rate of the 50 patients was $14.4 \%$, and the median OS time was 18 months. The correlations between the median OS time and the eight candidate factors (patients' age, operative procedure, operation time, tumor stage, occurrence of postoperative complications, preoperative CA 19-9 concentration, preoperative PNI, and postoperative PNI) among the 50 patients are shown in Table 3. The 50 patients were divided into 2 subgroups for each parameter according to the median values (patient age, operation time, and preoperative PNI). We found that only the postoperative PNI was an important risk factor for poor survival in patients with PDAC in the univariate and multivariate survival analyses (Tables 3, 4). Fig. 2 shows the OS curves of the patients.

\section{DISCUSSION}

Surgical resection is an essential treatment modality for

Table 2. PNIs between recovered group and non-recovered group

\begin{tabular}{lcccc}
\hline & $\mathrm{N}$ & \multicolumn{3}{c}{ Mean PNI $\pm \mathrm{SD}$} \\
\cline { 3 - 5 } & & Preoperative & 1 month after operation & 2 months after operation \\
\hline Recovered group & 23 & $44.6+6.9$ & $44.5+6.3$ & $48.6+6$ \\
Non-recovered group & 27 & $46.2+6.8$ & $35.8+8.8$ & $37.7+8.4$ \\
$p$ & & 0.365 & $<0.001$ & $<0.001$ \\
\hline
\end{tabular}

PNI, prognostic nutritional index; SD, standard deviation 
Table 3 Univariate survival analyses of 50 patients

\begin{tabular}{|c|c|c|c|}
\hline & $\mathrm{N}$ & $\begin{array}{l}\text { Overall MST } \\
\text { (months) }\end{array}$ & $p$ \\
\hline Patients' age & & & 0.315 \\
\hline Old $\geq 72$ years old & 27 & 19 & \\
\hline Young $<72$ years old & 23 & 15 & \\
\hline Operative procedures & & & 0.155 \\
\hline $\mathrm{DP}$ & 15 & 26 & \\
\hline PD+total pancreatectomy & 35 & 15 & \\
\hline Operation time & & & 0.214 \\
\hline Long $\geq 302$ minutes & 25 & 15 & \\
\hline Short $<302$ minutes & 25 & 22 & \\
\hline Tumor stages & & & 0.304 \\
\hline Stage I and IIA & 10 & 26 & \\
\hline Stage IIB and III & 40 & 17 & \\
\hline Postoperative complication & & & 0.536 \\
\hline Yes & 20 & 15 & \\
\hline No & 30 & 19 & \\
\hline Preoperative CA 19-9 & & & 0.364 \\
\hline High $\geq 37 \mathrm{U} / \mathrm{ml}$ & 40 & 17 & \\
\hline Low $<37 \mathrm{U} / \mathrm{ml}$ & 10 & 29 & \\
\hline Preoperative PNI & & & 0.264 \\
\hline High $\geq 46$ & 26 & 22 & \\
\hline Low $<46$ & 24 & 12 & \\
\hline PNI levels after operation & & & 0.003 \\
\hline Recovered group & 23 & 29 & \\
\hline Non- recovered group & 27 & 12 & \\
\hline
\end{tabular}

CA 19-9, carbohydrate antigen 19-9; DP, distal pancreatectomy; MST, median survival time; PD, pancreaticoduodenectomy; PNI, prognostic nutritional index

many potentially curable solid tumors. However, surgery induces alterations in patients' immune function. Surgical tissue damage promotes a strong inflammatory response and suppresses components of the immune system that protect against cancer. Thus, such surgical stresses can reportedly accelerate cancer growth and the risk of recurrence and may be correlated with poor patient survival. $^{13-15}$

PD with or without portal vein resection, DP with splenectomy, and total pancreatectomy with splenectomy are common operative procedures for PDAC. These operative procedures are associated with a long operation time and large blood loss volume, and in some cases, blood transfusions are required intraoperatively and postoperatively. Moreover, many patients develop postoperative complications. Thus, the surgical stresses of PDAC resection may be much greater than those of other gastrointestinal malignancies. Such surgical stress may be a poor prognostic factor for patients with resected PDAC.
Table 4. Multivariate overall survival analysis of 50 patients

\begin{tabular}{|c|c|c|c|}
\hline & $p$ & $\begin{array}{l}\text { Odds } \\
\text { ratio }\end{array}$ & $\begin{array}{l}95 \% \text { confidence } \\
\text { interval }\end{array}$ \\
\hline \multicolumn{4}{|l|}{ Patients' age } \\
\hline Old vs. Young & 0.401 & 0.731 & $0.351-1.519$ \\
\hline \multicolumn{4}{|l|}{ Operative procedures } \\
\hline $\begin{array}{l}\text { PD+total pancreatectomy } \\
\text { vs. DP }\end{array}$ & 0.841 & 1.087 & $0.481-2.457$ \\
\hline \multicolumn{4}{|l|}{ Operation time } \\
\hline Long vs. Short & 0.909 & 0.96 & $0.471-1.953$ \\
\hline \multicolumn{4}{|l|}{ Tumor stages } \\
\hline $\begin{array}{l}\text { Stage IIB and III vs. I } \\
\text { and IIA }\end{array}$ & 0.611 & 1.263 & $0.515-3.1$ \\
\hline \multicolumn{4}{|l|}{ Postoperative complication } \\
\hline Yes vs. No & 0.262 & 1.454 & $0.756-2.793$ \\
\hline \multicolumn{4}{|l|}{ Preoperative CA 19-9 } \\
\hline High vs. Low & 0.742 & 0.857 & $0.343-2.143$ \\
\hline \multicolumn{4}{|l|}{ Preoperative PNI } \\
\hline Low vs. High & 0.052 & 2.008 & $0.993-4.049$ \\
\hline \multicolumn{4}{|l|}{ PNI levels after surgery } \\
\hline $\begin{array}{l}\text { Non- recovered group } \\
\text { vs. Recovered group }\end{array}$ & 0.004 & 3.206 & $1.449-7.095$ \\
\hline
\end{tabular}

CA 19-9, carbohydrate antigen 19-9; DP, distal pancreatectomy; $\mathrm{PD}$, pancreaticoduodenectomy; PNI, prognostic nutritional index

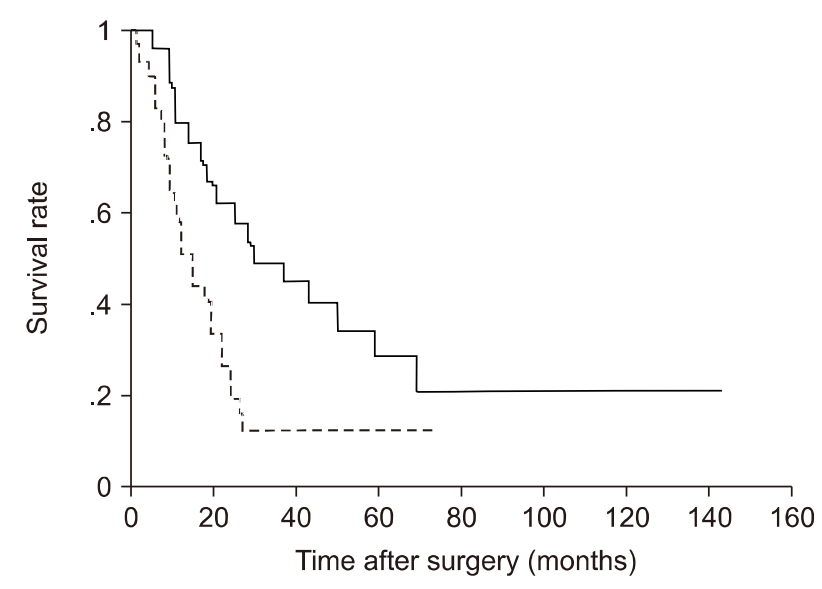

Fig. 2. Overall survival curves of 27 patients in the recovered group (solid line) and 23 patients in the non-recovered group (dotted line). The 5-year overall survival rate of patients in the non-recovered group $(7.4 \%)$ was significantly lower than that of patients in the recovered group $(24.3 \%, p=0.003)$.

The PNI represents patients' nutritional and immunological conditions. This index is designed to assess the nutritional and immunological status of patients. A low preoperative PNI has been used to estimate the occurrence of postoperative complications, and the postoperative PNI can be used to assess patients' recovery from operative damage. Murakami et al. ${ }^{16}$ recently reported the prog- 
nostic importance of comparison between the preoperative and postoperative PNI in patients with gastric cancer. Thus, in the present study, we focused on the changes in the PNI during the preoperative and postoperative periods as a new prognostic indicator for patients with PDAC. We analyzed the PNI of patients with PDAC at three time points (preoperatively and 1 month and 2 months postoperatively). We found that in 23 of 50 patients, the PNI at 2 months postoperatively recovered to the preoperative level, however, in the remaining 27 patients, the PNI at 2 months postoperatively did not recover. We also found that among several preoperative prognostic indicators in patients with PDAC, including the preoperative PNI, recovery of the PNI after the operation was a strong prognostic indicator in both the univariate and multivariate survival analyses.

Improvement of the nutritional status before and after the operation may improve the postoperative prognosis in patients with PDAC.

\section{ACKNOWLEDGEMENTS}

We thank Angela Morben, DVM, ELS, from Edanz Group (www.edanzediting.com/ac), for editing a draft of this manuscript.

\section{REFERENCES}

1. Onodera T, Goseki N, Kosaki G. [Prognostic nutritional index in gastrointestinal surgery of malnourished cancer patients]. Nihon Geka Gakkai Zasshi 1984;85:1001-1005. Japanese.

2. Nozoe T, Kohno M, Iguchi T, Mori E, Maeda T, Matsukuma A, et al. The prognostic nutritional index can be a prognostic indicator in colorectal carcinoma. Surg Today 2012;42:532-535.

3. Kato M, Hashimoto $H$, Noro $T$, Takahashi H, Hirashima $T$, Yamashiro A, et al. Pre and postoperative immunity in aged cancer patients-with special refference to the postoperative mehcl- lin-cefem resistant staphylococcus aureus (MRSA) infection and cell-mediated immunity. J Jpn Surg Assoc 1992;53:1033-1038.

4. Sagawa M, Yoshimatsu K, Yokomizo H, Yano Y, Okayama S, Yamada Y, et al. Pulmonary dysfunction function and poor nutritional status are risk factors for remote infections following surgery for colorectal cancer. J Nippon Med Sch 2018;85:208-214.

5. Ikeguchi M, Ashida K, Saito H. New prognostic indicator is useful for predicting the survival of patients with unresectable advanced colorectal cancer. Hepatogastroenterol 2015;62:61-64.

6. Li D, Yuan X, Liu J, Li C, Li W. Prognostic value of prognostic nutritional index in lung cancer: a meta-analysis. $J$ Thorac Dis 2018;10:5298-5307.

7. Luo Z, Zhou L, Balde AI, Li Z, He L, Zhen Wei C, et al. Prognostic impact of preoperative prognostic nutritional index in resected advanced gastric cancer: a multicenter propensity score analysis. Eur J Surg Oncol 2019;45:425-431.

8. Yamamoto T, Yagi S, Kinoshita H, Sakamoto Y, Okada K, Uryuhara $\mathrm{K}$, et al. Long-term survival after resection of pancreatic cancer: a single-center retrospective analysis. World J Gastroenterol 2015; 21:262-268.

9. Sato D, Tsuchikawa T, Mitsuhashi T, Hatanaka Y, Marukawa $\mathrm{K}$, Morooka A, et al. Stromal palladin expression is an independent prognostic factor in pancreatic ductal adenocarcinoma. PLoS One 2016;11:e0152523.

10. Greene FL, ed. AJCC cancer staging handbook: from the AJCC cancer staging manual. 6th ed. New York: Springer, 2002.

11. Li Z, Bai B, Ji G, Li J, Zhao Q. Relationship between ClavienDindo classification and long-term survival outcomes after curative resection for gastric cancer: a propensity score-matched analysis. Int J Surg 2018;60:67-73.

12. Choe JW, Kim HJ, Kim JS, Cha J, Joo MK, Lee BJ, et al. Usefulness of CA 19-9 for pancreatic cancer screening in patients with new-onset diabetes. Hepatobiliary Pancreat Dis Int 2018; 17:263-268.

13. Riedel B, Sloan E, Forget P. Long-term consequences of the acute neural-inflammatory stress response in the cancer surgical patient: new findings and perspectives. Int Anesthesiol Clin 2016; 54:58-71.

14. Gottschalk A, Sharma S, Ford J, Durieux ME, Tiouririne M. Review article: the role of the perioperative period in recurrence after cancer surgery. Anesth Analg 2010;110:1636-1643.

15. Horowitz M, Neeman E, Sharon E, Ben-Eliyahu S. Exploiting the critical perioperative period to improve long-term cancer outcomes. Nat Rev Clin Oncol 2015;12:213-226.

16. Murakami Y, Saito H, Kono Y, Shishido Y, Kuroda H, Matsunaga $\mathrm{T}$, et al. Combined analysis of the preoperative and postoperative prognostic nutritional index offers a precise predictor of the prognosis of patients with gastric cancer. Surg Today 2018;48:395-403. 